\title{
Iterative Design of MOVE: A Situationally Appropriate Vehicle Navigation System
}

\author{
Joonhwan Lee ${ }^{\dagger}$, Jodi Forlizzi ${ }^{{ }^{*}}$, and Scott E. Hudson ${ }^{\dagger}$ \\ Human-Computer Interaction Institute ${ }^{\dagger}$ and School of Design * \\ Carnegie Mellon University \\ 5000 Forbes Avenue, \\ Pittsburgh, PA, 15213, USA
}

\begin{abstract}
Drivers need assistance when navigating an unfamiliar route. In-vehicle navigation systems have improved in recent years due to the technology advances, but are sometimes problematic because of information overload while driving. To address the attentional demands of reading a map while driving, we have developed the MOVE (Maps Optimized for Vehicular Environments) in-car navigation display, which provides situationally appropriate navigation information to the driver through optimization of map information.

In this paper, we describe the iterative design and evaluation process that shaped the MOVE system. We describe early map reading and navigation studies that led to early designs for our system. We present a study on visual search tasks that refined the renditions used for the system. Finally, we present a study on the effectiveness of several variations of a contextually optimized route map visualization with a desktop steering system.

The result of this study shows that MOVE's contextually optimized navigation information can reduce the driver's perceptual load significantly. Our laboratory experiment shows that the total map display fixation time was decreased six-fold, and the number of glances to interpret the map display were decreased about threefold, when comparing the contextually optimized display to a static display.
\end{abstract}

\section{Keywords}

Maps; in-car navigation systems; visualization; human attention; perceptual optimization; dynamic displays

\section{Introduction and Motivation}

Many modern vehicles are equipped with in-vehicle navigation systems that utilize global positioning systems (GPS), digital maps, and automatic route calculation. Just entering a destination will typically generate an accurate route that is displayed to the driver, although the activity of entering a destination is not yet easy with current systems, especially while driving (Tijerina, Parmer et al. 1998; Burnett, Summerskill et al. 2004). Unlike turn-by-turn directions or traditional maps, these systems can track the vehicle's current location on the route, and can recalculate the route if a driver fails to follow it.

However, driving requires a lot of concentration and can be easily distracted by other in-vehicle activities (such as chatting with passengers, talking over the cell phone, manipulating instrumental panels, or changing radio stations). This can sometimes increase the hazards of driving. Not surprisingly, in-vehicle navigation systems, while offering considerable advantages over for example paper maps, can present similar issues. In particular, current navigation systems typically do not carefully consider a driver's cognitive load and attentional state - delivering all information in the same way regardless of context. This paper considers the design of the MOVE (Maps Optimized to the Vehicular Environment) display technique (Figure 1). The MOVE display is designed to carefully tune how much attention is demanded by elements of the presentation so that the most important information in the current context remains highly salient, while reducing the distraction of surrounding information. However, it does this without eliminating the cues that drivers typically use to maintain their mental model of where they are, and when features of interest will be approaching. 


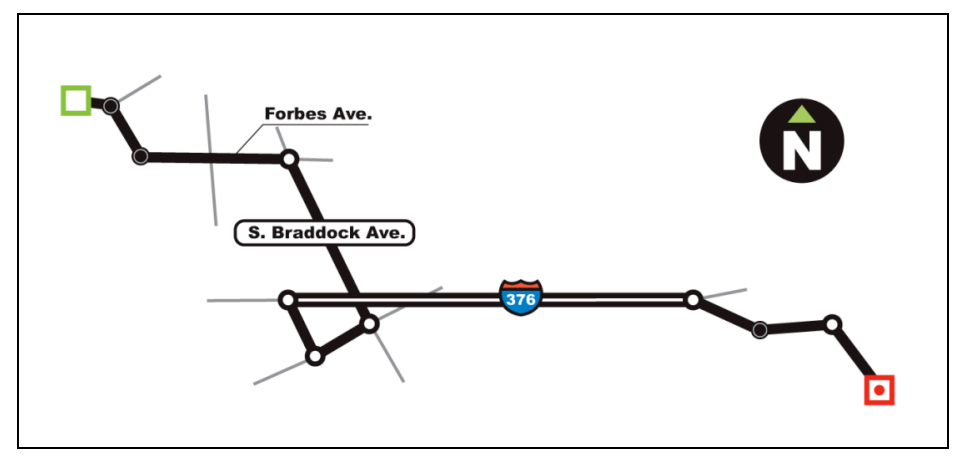

Figure 1. A sample of the MOVE display

A few researchers have reviewed in-vehicle navigation systems (Ross and Burnett 2001; Llaneras and Singer 2003). We initially informally reviewed several in-vehicle navigation systems to discover their design issues. Three of them are pre-installed systems (Nissan, Honda, and Mercedes Benz), and two of them are the most popular after-market products (Garmin and Magellan).

Both pre-installed systems and after-market systems employ similar presentation styles. Usually, a vehicle location cursor is placed in the center of the display and the route map scrolls down as vehicle moves forward. Most systems use heading-up as a default presentation style, but users can also switch to north-up style if needed. Displays on current in-vehicle navigation systems are often modeled after paper maps, and have similar visual complexity. Therefore, some research has suggested that, due to the visual complexity of map data, it is important to limit the amount of information presented to the driver when they are driving (Streeter, Vitello et al. 1985; Labiale 1990; Parks, Ashby et al. 1991), and even suggested that map information only be presented when the vehicle is stationary (Michon 1993; Ross, Vaughan et al. 1995). Because relatively small displays are often used, such systems almost always allow the user to zoom into areas of interest in the current driving context to display an affordable amount of information to a driver. However, this tends to provide a myopic view and reduces global route tracking cues. Further, even with views of only a small area, drivers still need to expend a significant amount of attention in order to get right information out of the display. One solution attempted in current systems has been the use of simple iconic depiction of important sections or turns (Figure 2).

Differences between systems can be found in the use of simple icons. Since pre-installed in-vehicle navigation systems usually have a bit wider screen than after-market products, their displays are often vertically divided into two columns. One column provides an overview of the route, while the other shows a simple iconic depiction. After-market products show a simple iconic depiction of important turns as a small pop-up window, or by replacing the main display for a few seconds with an iconic depiction.

While dramatically reducing the associated visual search task, iconic representations eliminate nearly all the context information that a driver normally uses to maintain a mental model of their location. Further, it can also eliminate the cues needed to choose between several physically or temporally close alternatives, making it hard to match the display to the current driving context.

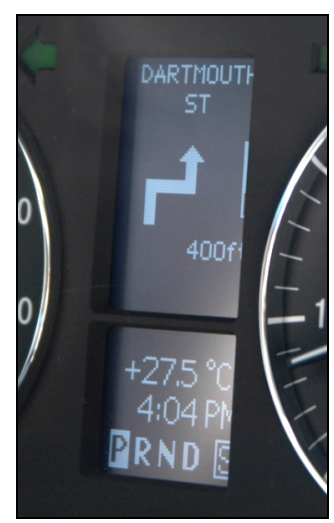

Figure 2. Iconic form of route 
In contrast, the MOVE display seeks a balance which makes the information most likely to be important in the current context easy to acquire from the display, while maintaining global context. Our overall goal can be simply stated as reducing the time a driver needs to spend looking away from the road to use an in-car navigation system. We have previously shown (and will summarize again here) experimental results which show that the system reduces six-fold the total time spent looking away from an attention saturating laboratory task analogous to driving.

To achieve this result, we employed a thorough HCI design process which intermixed more intuitive methods from the discipline of design with testing and analysis methods with roots in the behavioral sciences. In particular, we began work with needs finding, looking at issues of the current systems to be addressed in our research through literature review and structured observation of users. We then developed our initial idea of the system and sketches of possible display components. A study was later undertaken to more rigorously measure the perceptual effects of these concepts. These measurements validated our initial design concepts and allowed us to develop principles and guidelines for the system. A full design and accompanying prototype was created, and the effects of our approach were again carefully measured. The remaining sections of this paper will follow the steps outlined above and provide more detail for each.

\section{Background on Maps, Map Reading, and Navigation}

A map is an abstracted two-dimensional representation of a three-dimensional reality which is rich in detail. As such, all maps are based on the use of abstraction. Some forms of abstraction act by simply omitting information which is less relevant to the task at hand. Other forms of abstraction may retain (partial) information, but simplify or distort it to make it more discernable in a given task context (Monmonier 1996).

The London Underground maps of the 1920s (Figure 3a) and recent (Figure 3b) are outstanding examples of how the use of abstraction can improve the legibility of a map rendition. Figure $3 \mathrm{a}$ is a depiction that remains true to the curvature of the land, employing significant detail and accurate paths for each underground line. In contrast, Figure $3 \mathrm{~b}$ abstracts away detail, presenting routes in a schematic rather than realistic fashion. In Figure 3b, the reduction of detail and the distortion of the route relative to the city's geometry make it possible to focus on the most relevant information.

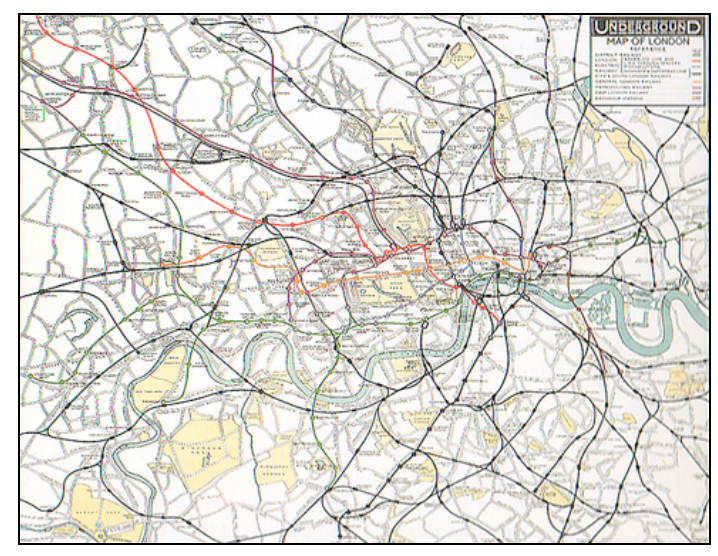

(a)

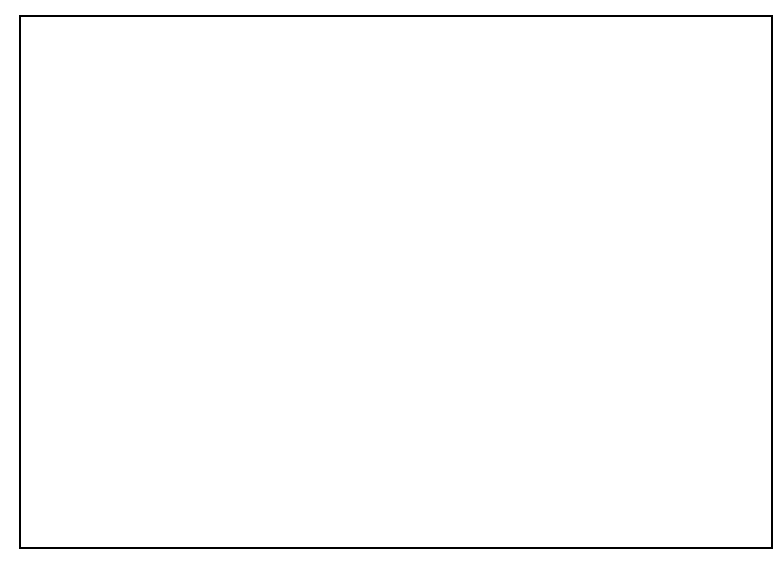

(b)

Figure 3. (a) London Underground Map (1920's), (b) London Underground Map (recent)

\subsection{Map Generalization}

Abstraction techniques such as those used in the London Underground map have been used by cartographers for years. Gradual refinement of this technique has resulted in the process of map generalization. Monmonier (Monmonier 1996) has categorized the generalization process into several 
steps. Our design has been guided by at least 5 of these: selection, simplification, displacement, smoothing and enhancement. Features are selected in a map to support the specific task of the map. Selected features will be more prominent than other features to draw more of the user's attention. Simplification reduces detail from map features. For example, in the London Underground map, the angularity of lines has been reduced by removing points along the path. Displacement avoids possible graphical overlap or clutter by mediating the size and location of each feature. Smoothing also reduces detail. In contrast, enhancement adds details to the selected features to convey more information when essential to the task.

The LineDrive system (Agrawala and Stolte 2001), shown in Figure 4, is a good example of how abstraction can be successfully applied to a static route map. Unlike many other Internet based route maps, LineDrive uses the generalization technique to create an abstract route map. For example, many online map services use a constant scale factor to generate a traditional map. LineDrive uses various scale factors for each road segment, based on the importance of the segment. Unnecessary features are also removed from the map. Distortion, simplification and other abstraction techniques are also used based on the importance of the segment in the route. Landmarks are used sparingly. All of these techniques make the map easy to read, and reduce the driver's perceptual load while driving.

The LineDrive system has been inspiring to our research. However, because of its static nature, LineDrive cannot provide an optimized route that is sensitive to the context of the driver. A contextually optimized route display could provide appropriate route information to a driver through awareness of a driver's situation at various times during the drive. For example, the system could display more or less detail based on the current location in the route or speed of a car.

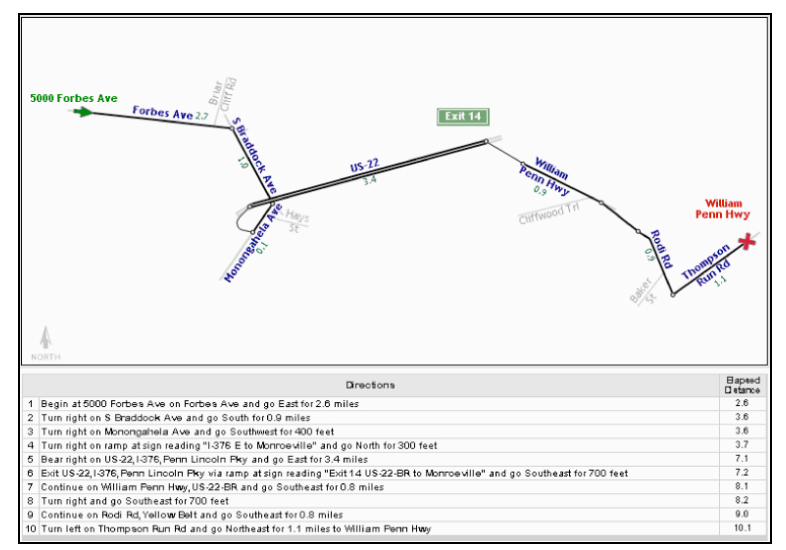

Figure 4. LineDrive

\subsection{Navigation}

The goal of navigation is to achieve movement through space. It is generally assumed that mental representation of a geographical area is based on three kinds of knowledge: landmark, route, and survey (Wickens and Hollands 2000). Landmark knowledge is a representation that includes the appearance of prominent landmarks in the region - gas stations, tall buildings, or parks. Landmark knowledge is gained by direct experience in the environment, and is highly relative to the individual viewer. Route knowledge is proceduralized knowledge about how to get from one place to another. Landmarks are usually incorporated into route knowledge. Route knowledge is also centered on the individual, but does not supply a great deal of information for learning more about the route. Survey knowledge is abstract, spatial knowledge that allows the individual to draw an accurate map of the environment. It usually represents geographical knowledge that has been generalized over many experiences, and so is more objective than either landmark or route knowledge. Individuals most commonly gain knowledge in the order of landmark, route, and survey information when repeatedly visiting an area.

Several researchers have introduced models for how navigation occurs during a driving task. Michon uses three different levels: strategic, maneuvering and control (Michon 1985; Ross and Burnett 2001). The driver plans a route at the strategic level, maintains their position on the route at the maneuvering level, and controls the vehicle (e.g., accelerate or decelerate) at control level. Burnett extended Michon's model by 
integrating a driver's requirement and goals for a navigation system. The model has six overlapping stages: trip planning, preview, identify, confirm, trust, and orientation (Burnett 1998; Ross and Burnett 2001). In the trip planning stage, the driver will specify a destination and plan a route. In the preview stage, sub-goals will be established by assessing perception of remaining time and distance to the next maneuver, and building a mental picture and preparatory knowledge of the maneuver. In the identify stage, the driver will identify the direction to travel, control suitable speed of the vehicle, and establish correct positioning of the vehicle on the road. In the confirm stage, the driver will verify whether the correct maneuver has been made. In the trust stage, the driver will gain assurance that the correct route is being driven. Finally, in the orientation stage, the driver will remain aware of their current location in the entire route, especially in relation to final destination (Ross and Burnett 2001).

\subsection{Map reading}

A map is one of many potential representations of a space that the viewer may draw upon as an aid to decision-making. A particularly valuable approach is that of MacEachren, who seeks to combine both lowlevel perceptual theories, such as those derived from Gestalt psychology, with higher-level cognitive processes, such as those derived from an information-processing theory of cognition, into a comprehensive theory of how maps are read and interpreted (MacEachren 1995). These encompass both bottom-up (sensory stimulus driven) and top-down (goal or cognitively driven) approaches, although MacEachren reports a debate in the literature as to how much the bottom-up approach plays a part in map reading. MacEachren's view of map comprehension is based on three stages of processing: a precognitive visual array, where shapes, edges, and boundaries are detected; a 2.5D sketch, or visual description, where a visual description is held in short-term memory, and the representation is initially mediated with the viewer's existing knowledge; and finally, a representation that holds meaning and generates knowledge for the viewer.

\subsection{Prior knowledge and preferences}

As people become more familiar with an environment, they become more confident in their own cognitive representations and their dependency on external aids such as landmarks, written or verbal directions, and signage decreases.

Golledge maintains that cognitive maps, or representations of prior knowledge about a route, can be defined in two ways: as representations of analog maps that are retained in memory, or as metaphorical representations that enable a person to act as if he has access to a map (Golledge 1999). According to Golledge, the term cognitive map implies deliberate and motivated encoding of environmental information which can be used to determine where one is at any moment, where specific encoded objects are in surrounding space, how to get from one place to another, or how to communicate spatial knowledge to others.

Landmarks play an important role in cognitive maps because they might have a peculiar form, or sociocultural significance. In addition, unremarkable environmental attributes may attain salience for particular individuals, because they are tied to one's history (for instance, a place of work or the home of a childhood friend). Landmarks are hierarchically organized in cognitive maps based on significance and location.

As people stray from their initial paths in an environment, they integrate new environmental information into the existing cognitive map and eventually progress from route-based knowledge to survey knowledge. Since certain routes are better learned than others, survey representations are often incomplete, distorted, or incorrect. However, survey knowledge has been shown to be more reliable than route knowledge.

\section{Needs Finding Qualitative Studies}

In order to understand additional factors that influence a driver's ability to navigate within a space, and more directly inform our design process, we conducted a four-month qualitative research study. We wanted to understand how people read maps, make directions, and use directions while driving. Backgound 
research on this issue revealed that people tend to ask other people to provide a route, rather than study maps, and that people tend to a route described by prominent features of the environment, rather than travel time or distance (Streeter, Vitello et al. 1985; Denis, Pazzaglia et al. 1999; Burnett 2000). Studies also show that navigation with well-described routes yield significantly lower error scores than navigation with poorly described routes, and that skeletal descriptions yield scores similar to detailed descriptions (Denis, Pazzaglia et al. 1999). We therefore theorized that personal preferences for navigation, particular criteria of existing directions and maps, and prior knowledge of route would be the most significant factors. We drew inspiration from research on navigation, on map reading, and on the role of prior knowledge in helping people find their way to a destination to provide an overarching structure for our research and to generate themes and protocols for our studies.

Fifteen participants ranging in age from 20-54 performed a series of three pilot studies on the topic of navigation. In the first study, we wanted to understand how participants give written or drawn directions to familiar, not-so-familiar, and unfamiliar places. We also wanted to understand what criteria are valued about printed maps and atlases. In the second study, we wanted to understand how generating directions differs when one is driving, navigating, or creating a route for a third party. In the third study, we wanted to understand how preference for using directions might differ when people were navigating to an unfamiliar destination on a small street as opposed to an unfamiliar destination on a large street that transpires a number of miles. For that study, we limited the resources available to LineDrive computer-generated directions (Microsoft 2005).

We found that drivers continually monitor their location relative to a given route, possibly involving a map or some representation of the route, and occasionally change route if circumstances warrant. Road maps can be helpful to drivers; line-by-line directions were found to be somewhat less helpful.

When navigating, we observed that drivers break the route into smaller steps, or subgoals. The steps may be as small as those in line-by-line directions, or they may be made up of schematized sections of the route that drivers already know (for example, home to the on-ramp of the nearest major highway). To find the way from goal to goal, drivers rely mostly on information about landmarks, paths (important streets), and nodes (intersections of two important streets). For a number of reasons, some landmarks are more salient than others, but they are used to guide the journey, acting as both confirmation points and ways to mark the next important turn on the route. Landmarks are also used for error prevention, and to reorient oneself to the route when lost.

When asked to create a representation of the route, participants often made two versions of maps: a version for other people driving who did not know the route (Figure 5), and a version that they would prefer to use for themselves (Figure 6). Abstracted, flattened, and simplified representations were consistently produced. When following directions, landmarks were salient in the following order: neighborhood, known (usually large) street, orientation of destination street (parallel or perpendicular to a known street), number on destination street, right or left side, and nearby landmark. We routinely observed participants seeking landmarks in such a focused way that they would miss a landmark if they had not found the prior one. 


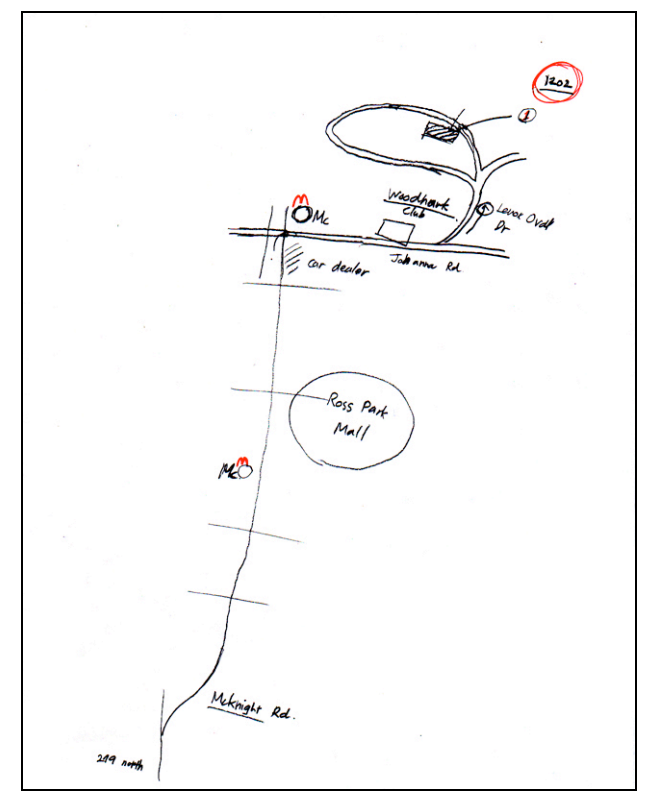

Figure 5. A representation generated for other people driving who did not know the route. Landmarks play an important role here, and details have been abstracted away.

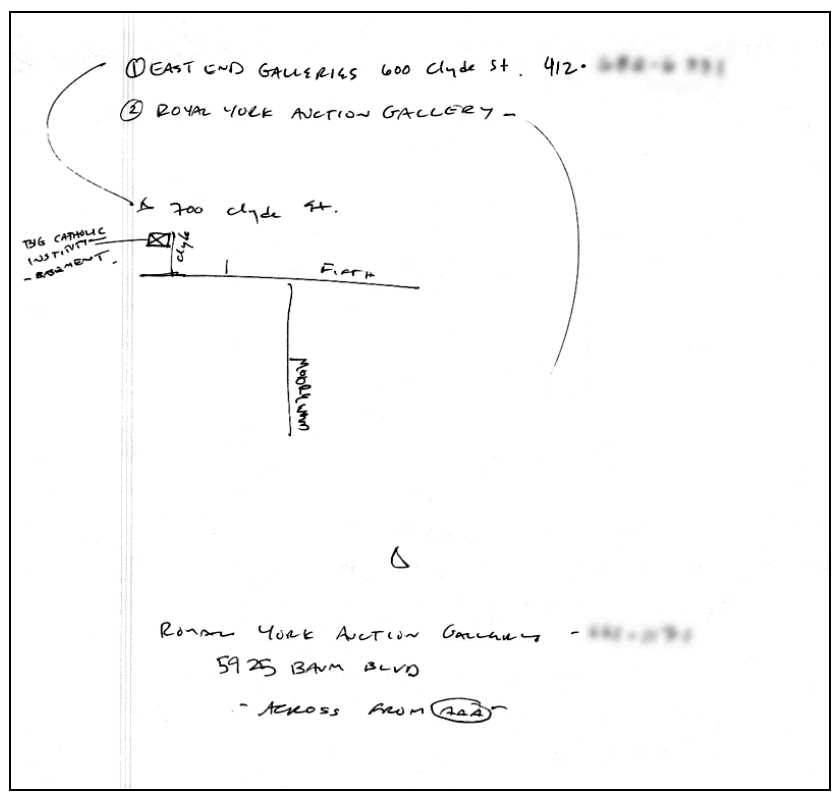

Figure 6. A representation generated for a participant to use himself. Neighborhoods play an important role in this representation; arrows mark the exact location of the destinations and provide important annotations.

\section{Preliminary MOVE Design}

The navigation study described above provided information about how people plan and visualize a route. Many people use abstraction in their visualizations and selectively place information on the map based on their own judgments. Figure 7 is a good example of a route representation. Figure $7 \mathrm{a}$ is a hand-drawn route map to be used to navigate to a local shopping mall. Much of the pertinent information such as cross-streets and road labels are left off the map. Additionally, the actual length, shape, or direction of a road section is arbitrarily distorted. However, the main critical roads on the route are presented as thicker lines and junctions where critical turns need to be made are represented with details. Some landmarks, cross-streets, and road labels are selectively placed.

Making use of the abstraction techniques used in these hand-drawn route maps, we produced preliminary concept sketches for the MOVE design as illustrated in Figure 7b. This MOVE route map uses turns as a main unit of representation and removes unimportant roads from the map. Additionally, unimportant labels are removed from the map and each junction and road is presented with a different style of rendition based on its importance in the current context. We also designed an initial set of symbols to be used in the MOVE display - for example, node symbols for major turns, various types of cross-street markers, road labels, and other symbols such as the vehicle position mark, route start and route end. 


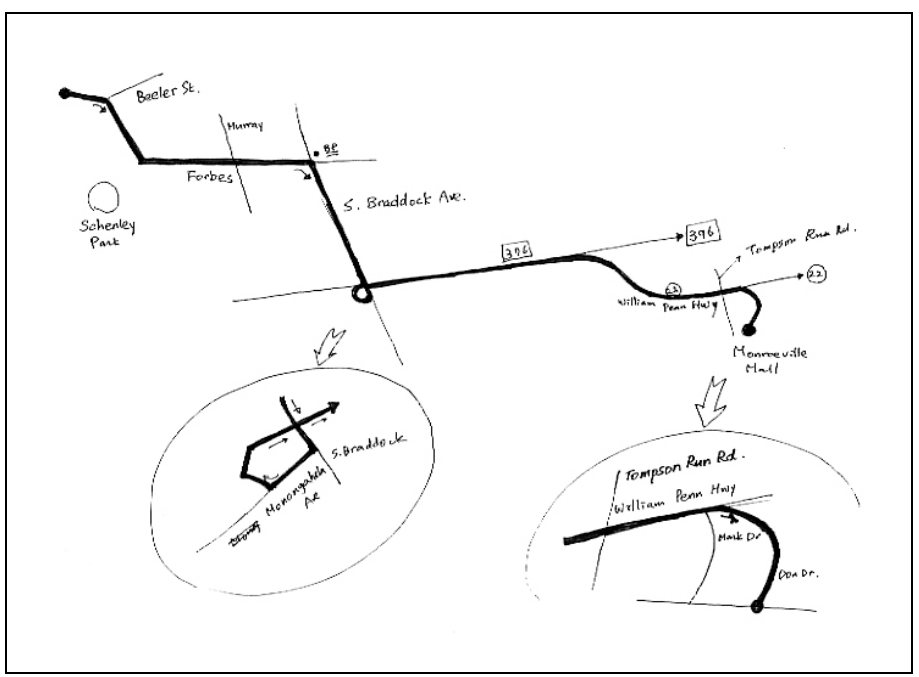

(a)

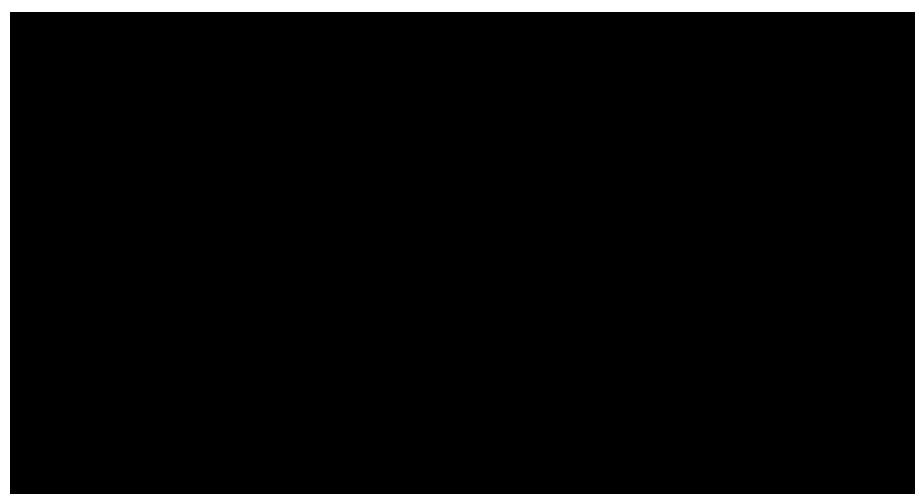

(b)

Figure 7. Hand-drawn map and early sketch of the MOVE display

Our early exploratory interviews and designs led us to the high level design principle of optimizing the balance between the positive communicative benefits of selected map elements and the potential negative effects of distraction. In particular, our goal would be to produce for each situation a map which helped the user maintain the context of where they were in the route, make the information most important to that situation easy to perceive, and minimize the distraction caused by other information. A more detailed refinement of this principle will be described when discussing the final design below.

\section{Visual Search Study}

In order to obtain a detailed understanding of the perceptual effects of the renditions we had devised in our initial sketches, we performed a study of how particular renditions affect visual search, both when they are the target of the search (providing positive communicative benefit), and when they serve as distraction from the target (inducing a negative effect). Quantitative information from this study allows us to place our final design on a firm empirical footing and grounds the optimization tradeoffs it makes.

Finding targeted information in a map is a visual search task. Researchers have found that there are two major types of visual search mechanism. The first type are top-down mechanisms which are goal driven and implement our cognitive strategies (Conner, Egeth et al. 2004). The second type are bottom-up mechanisms. Bottom-up mechanisms are thought to operate on raw sensory input, rapidly and involuntarily shifting attention to salient visual features of potential importance. Many scientists have pointed out that neither type of mechanism works in isolation in a particular situation; instead, the mechanisms work 
together interactively. Typically, bottom-up mechanisms act early in the visual perception process, and then top-down mechanisms take over, generally within a time on the order of 100 milliseconds. (Conner, Egeth et al. 2004).

Within bottom-up mechanisms the concept of pop-out (Baldassi and Burr 2004) is an important one which has implications for visual design. Pop-out is a bottom-up drawing of attention to an object, which occurs when an object within the visual field is distinctive along some visual dimension, for example, possessing a distinctive color or brightness when compared with other objects in the field. Prior studies have identified a range of visual features which can induce pop-out effects, including color, brightness, movement, direction of illumination, distinct curvature, and tilt (Beck 1982; Julesz 1984; Treisman 1986; Treisman 1998). Notably however, size has not been shown to strongly induce this effect. (Baldassi and Burr 2004)

We would expect the same visual search phenomena to apply to the specific case of a map reading task. When a driver looks for a target, they will generally have in mind what they are looking for (e.g., an indication of where the next turn is, or how far from the next turn they currently are). Correspondingly, we would expect to see baseline performance effects related to goal directed top-down perception, modified when pop-out effects occur. In our case, the effect of bottom up pop-out effects will be positive for objects which are the target of the user's search (they will tend to lead to finding the target object faster) but negative for objects which are not the target of the user's search (which will become more distracting and slow down finding the target object). The details of how large these effects are relative to one another and which symbols induce pop-out effects in relation to others is important to determining which symbols should be selected and where they should be placed. To uncover these details, we undertook a visual search study using the specific renditions proposed for the MOVE system.

\subsection{Experiment Overview}

In our study, participants were asked to find target information from within a display. We measured their reaction time and error rate for this task. A map stimulus with a road depiction containing several symbols was presented (Figure 8). Participants were verbally prompted to select a target rendition from a map stimulus, indicating the position of the rendition by pressing a keyboard key. We analyzed response time for renditions treated both as search targets and as distracters, considering all rendition pairs in order to understand their performance effects in light of both top-down and bottom up (pop-out) effects. In the remainder of this section, we consider the experiment in more details and present its results.

\subsection{Participants and Experimental Procedure}

Twenty people from our university community participated in the study - 12 females and 8 males aged 18 to 33. The study was carried out in a lab setting under typical office lighting. After signing a consent form, subjects read simple instructions describing the overall process of the study, saw an overview of the renditions that the participants would see during the study, and were asked to perform selections as rapidly as they could. After becoming familiar with the renditions, they were presented with an example session of the study (employing 5 randomly selected study tasks) designed to give overall understanding of the study task. Finally, subjects completed a series of timed trials that formed the body of the study.

During the study trials, the participants were presented with a voice prompt using a pre-recorded female voice, indicating which symbol they should select. For example, before Figure 8 was presented, a participant would hear "East Avenue" while being presented with a blank screen. Immediately after the voice prompt, a visual stimulus would appear. Once the correct rendition was found, the participant indicated its position in the display by pressing 1,2 or 3 on the keyboard. Trials were repeated until every map stimulus was presented.

To record reaction time, the experiment software started a timer when the visual stimulus was placed on the screen and stopped when a participant pressed a key. Reaction time was recorded in milliseconds. We also recorded error responses. Error rates were extremely low and do not allow any useful distinctions to be made between the renditions, and thus will not be considered further. 


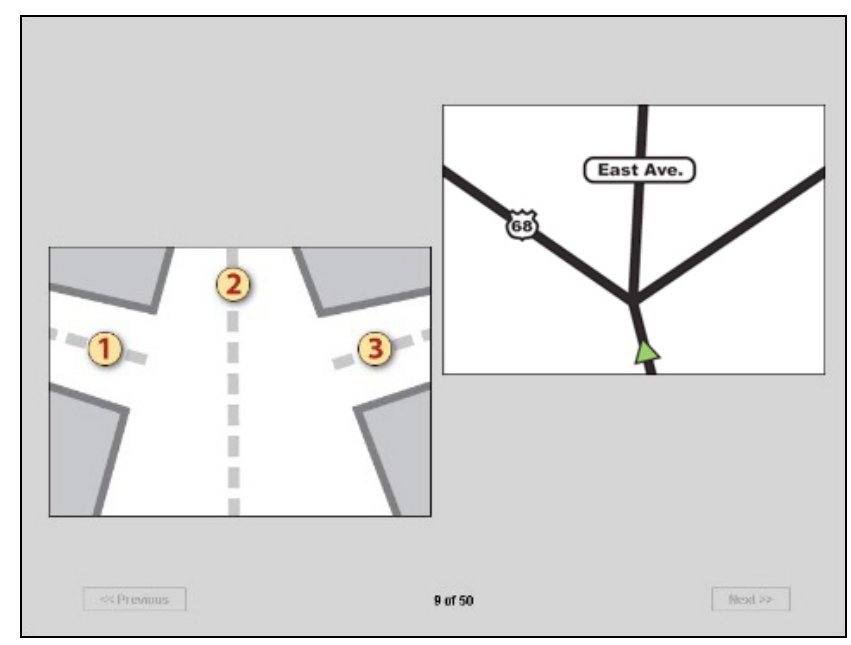

Figure 8. Search task screen

\subsection{Stimuli}

The stimuli were generated based on 13 renditions chosen from our earlier MOVE sketches (Figure 9). These included six different node (or intersection) renditions, five different forms of road labels, and two other renditions (route start and route end). In addition to this, as a check of our stimulus manipulation we also included an extra rendition we expected to be highly salient - a McDonald's logo (Table 1). To create the stimulus for each trial, we selected two renditions out of the 14 and placed them in two of three positions. Three different road renditions were employed. For each trial, one rendition was designated as the target (and the other was a distracter). Trials covered every target-distracter pair (but did not present a rendition paired with itself) for a total of $182(14 \times 13)$ pairs each presented once for each road type for a total of $546(182 \times 3)$ trials. The placement of selected renditions in the road positions (left, right, and center) and ordering of trials was randomized.

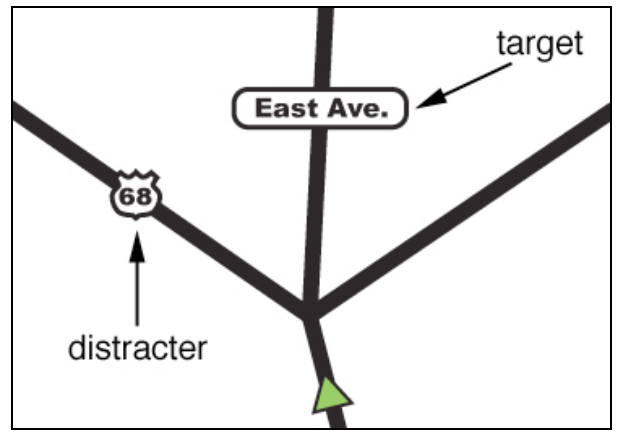

Figure 9. Stimuli - target-distracter combination

\begin{tabular}{|c|c|c|c|c|}
\hline A & B & C & D & E \\
\hline $\mathbf{O}$ & $\mathbf{O}$ & $\mathbf{O}$ & $\mathbf{O}$ & (1) \\
\hline F & $\mathbf{G}$ & $\mathbf{H}$ & $\mathbf{I}$ & J \\
\hline (1) & $\mathbf{3 6}$ & $\mathbf{6 8}$ & $\mathbf{8 3}$ & West Ave. \\
\hline K & $\mathbf{L}$ & $\mathbf{M}$ & $\mathbf{N}$ & \\
\hline East Ave. & $\square$ & $\mathbf{0}$ & M & \\
\hline
\end{tabular}

Table 1. Selected renditions for the study 


\subsection{Results and Discussion}

To understand the base salience of each rendition, we initially compared the mean reaction time for each rendition alternative when used as a target (across all distracters). As expected, the McDonald's icon was highly salient - its distinctive shape and color scheme makes it likely to induce pop-out effects when combined with the other symbols. (We have eliminated this rendition from the remainder of the analysis to avoid skew).

To help simplify target reaction time results, it is useful to group the renditions into symbolic and semantic categories. A symbolic rendition conveys its meaning through shape, while semantic renditions contain information conveyed through text and/or numbers. The more detailed road signs in our experiment (renditions $\mathrm{G}, \mathrm{H}, \mathrm{I}, \mathrm{J}$, and $\mathrm{K}$ ) are semantic, while the remaining renditions (A, B, C, D, E, F, L, and M) are symbolic. We also analyzed a more detailed set of categories: semantic text $(\mathrm{J}, \mathrm{K})$, semantic numbers $(\mathrm{G}$, $\mathrm{H}, \mathrm{I})$, complex symbols (B, D, F, M), simple symbols (A, C, E, L), colored (G, L, M), black and white (A, $\mathrm{B}, \mathrm{C}, \mathrm{D}, \mathrm{E}, \mathrm{F}, \mathrm{H}, \mathrm{J}, \mathrm{J}, \mathrm{K})$, and finally with respect to the size of each rendition: large $(\mathrm{B}, \mathrm{D}, \mathrm{F}, \mathrm{J}, \mathrm{K})$, medium (G, H, I, L, M) and small (A, C, E). (Table 2)
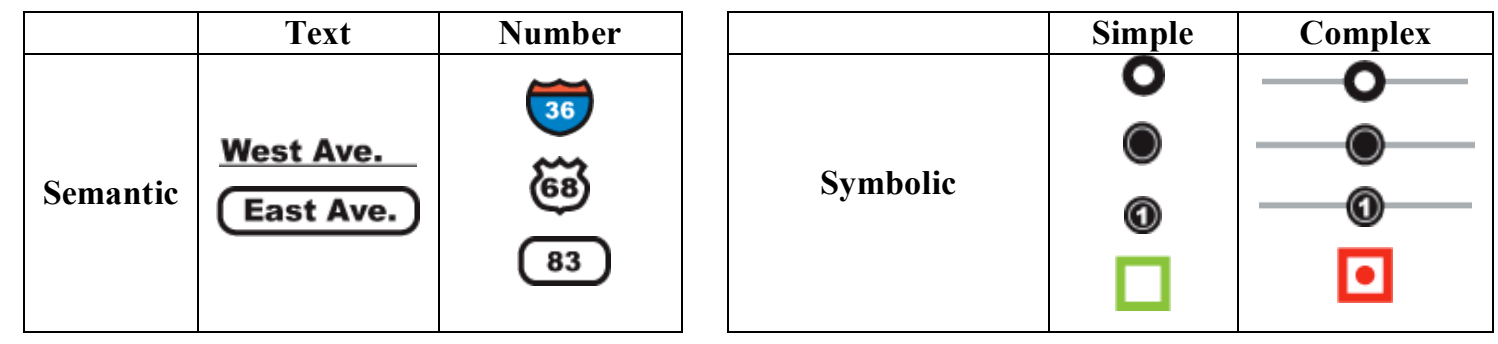

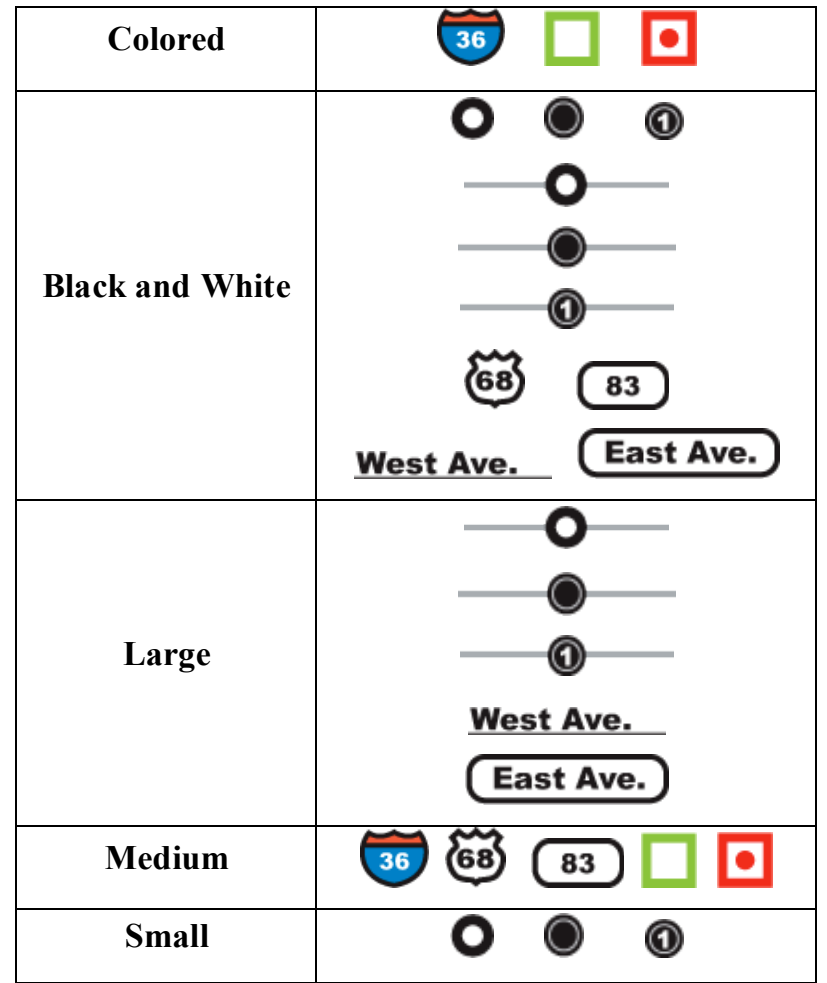

Table 2. Categorized renditions 
First, we compared the mean reaction times of semantic and symbolic renditions. As Figure 10 indicates, participants had faster reaction times when searching for semantic renditions. This result was statistically significant $(t(19)=-6.24, p<0.01)$, and could imply that participants may be able to more quickly interpret the meaning of semantic renditions. Comparison of finer semantic subcategories shows that reaction time for semantic text was the fastest, followed by semantic numbers, simple symbols and complex symbols, and that these results were all also statistically significant $(\mathrm{F}(3,57)=39.11, p<0.01)$. (Figure 11)

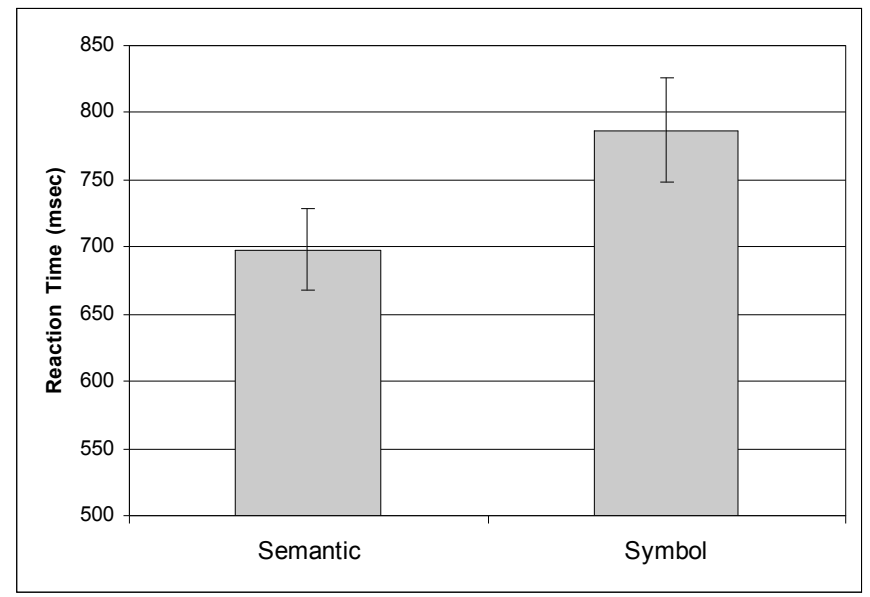

Figure 10. Semantic vs. Symbol

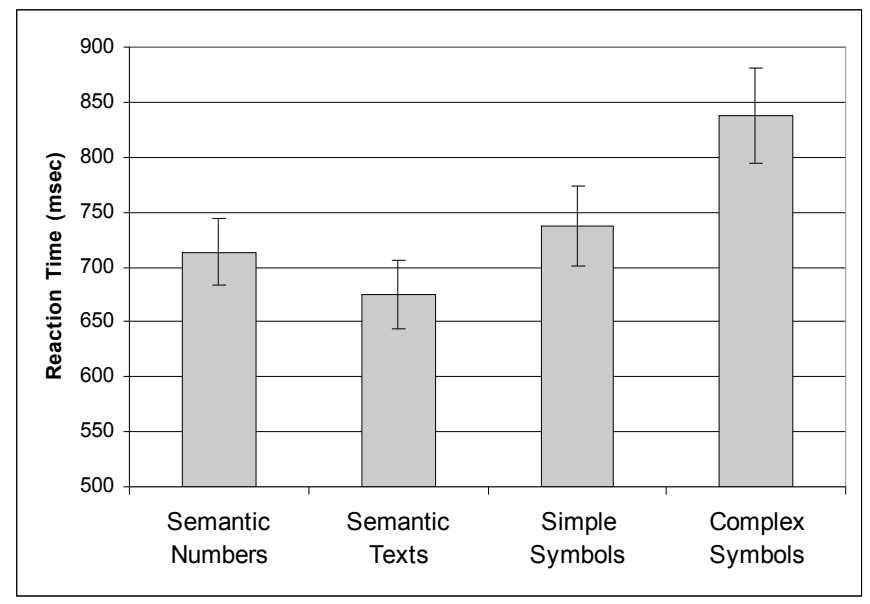

Figure 11. Semantic Numbers vs. Semantic Texts vs. Simple Symbols vs. Complex Symbols

A second set of comparisons allows us to explore the effects of renditions when they serve as distracters. Here we made a comparison of target-distracter combinations: Semantic(T)-Semantic(D), Semantic(T)Symbolic(D), Symbolic(T)-Semantic(D), and Symbolic(T)-Symbolic(D). Consistent with the results from simple mean reaction times, Figure 12 indicates that reaction time was faster when semantic renditions were involved. Further, data shows that if the target and distracter were of the same rendition type, then reaction time was increased. The result is statistically significant. $(\mathrm{F}(3,57)=35.10, p<0.01)$ 


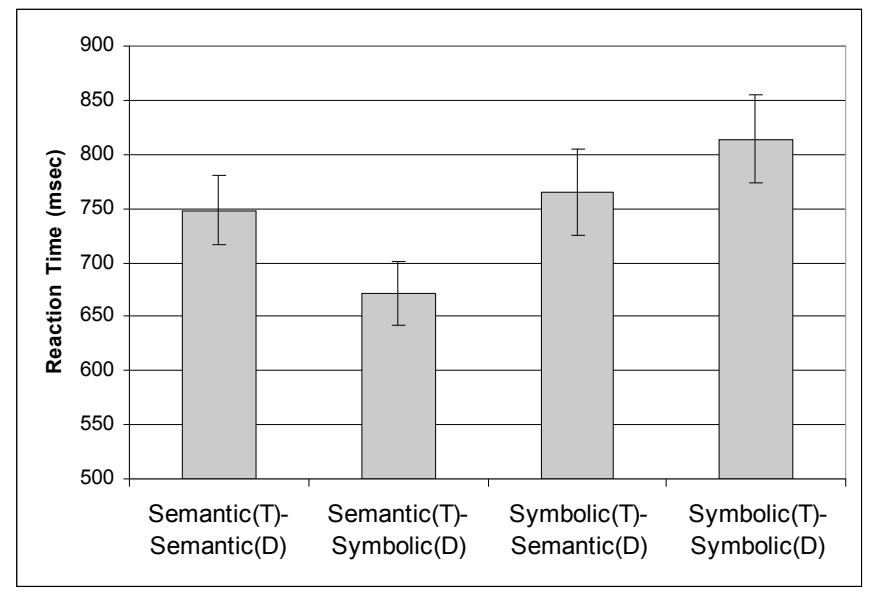

Figure 12. Semantic(T)-Semantic(D) vs. Semantic(T)-Symbolic(D) vs. Symbolic(T)-Semantic(D) vs. Symbolic(T)-Symbolic(D)

Prior work (Goldstein 2002) has found that if clear pop-out occurs in a search task, reaction time is consistently fast no matter how many distracters are present. This activity is characteristic of a bottom-up search. As an exploration of this effect we considered reaction times when black and white and contrasting colored stimulus were used together. In comparing mixed stimulus with all black and white stimulus we found that reaction times for colored targets were significantly faster $(t(19)=7.79, p<0.01)$ (Figure 13).

In contrast, we also determined that rendition size was not effective as a pop-out. Differences among the three different rendition sizes were not statistically significant $(\mathrm{F}(2,38)=1.423, p=0.25)$. This is in accordance with other research on bottom-up searches (Julesz 1984; Treisman 1986; Treisman 1998; Baldassi and Burr 2004). Additionally, we did not find any interaction effects between road types, semantic renditions, and symbolic renditions $(\mathrm{F}(2,38)=0.6, p=0.55)$.

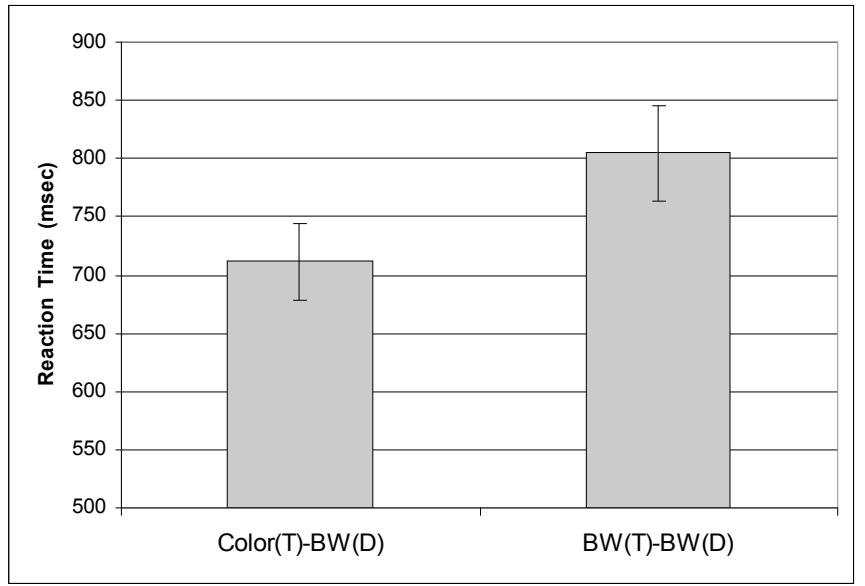

Figure 13. Pop-out effect

\section{Detailed Design Principles}

Based on our preliminary review of cartographic design, research on navigation, and research on rendition types, we generated detailed design principles for the MOVE system. The overall purpose of the system is to minimize perceptual load while driving. Three principles uphold this purpose. First, specific choices for the display should reflect the likely importance of the information in the current situation. Second, the navigational information should be presented in an abstract manner, while considering the driver's current context. Finally, the system should present dynamically optimized information so that the driver's direct 
interaction with the system can be minimized. In the next sections, we consider the specifics of each of these principles.

\subsection{Using Importance Differences}

In any given situation, not all information in the display will be of equal importance (or equally likely to be the target of a visual search). By using the most salient and attention demanding display elements only for the likely high importance items, while lowering the salience or even removing others, we can expect to achieve a perceptually efficient display.

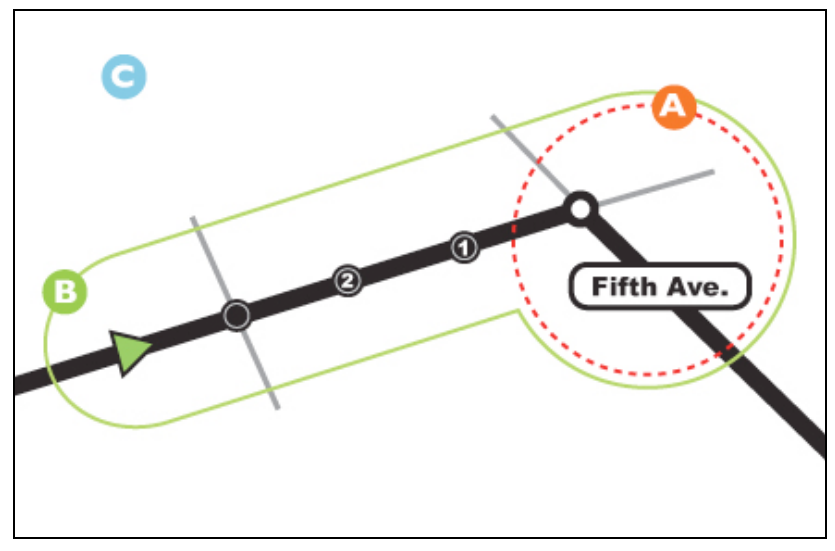

Figure 14. Areas of different importance

Figure 14 shows a depiction of how different areas of the display are assigned differential importance. The display is divided into three regions. Region $\mathrm{A}$ is what is most important to the driver - the information about the next turn. Region B is the next most important information, the area surrounding the current position of the vehicle, working forward to the next turn once it is close enough. Region C encompasses the remaining surrounding area (where minimal or no renditions are used).

The results of the study described in the previous section provide information about the choice of renditions with respect to the importance of the regions they fall within. Semantic renditions should be used primarily for important areas (region A and sparingly in region B), while symbolic renditions should be used in areas that need less visual salience (region B and occasionally in region C). Finally, pop-out inducing renditions should be used very sparingly and only in locations of most likely current interest.

\subsection{Abstraction}

A second high level design principle involves the use of abstraction and generalization techniques. When designing MOVE, we categorized the map generalization process into the following five aspects.

\subsubsection{Map Feature Selection}

A route consists of several segments that the driver will traverse during the course of the route. Various map elements exist along the route, but not all of them can be presented on the display. Usually, drivers pay attention to the road segment they are currently traveling on. Other sections on the route or nearby, including landmarks such as rivers, parks, municipal boundaries, and other map features are not important unless they play a key role in navigating the route. For example, a gas station near the next turn becomes important as a vehicle is approaching the turn because it could be used as a landmark or a milestone. Feature selection is a process which, in a similar way, determines what features should be included on a particular map. Once selected for inclusion, a rendition for each feature is selected based on its importance (as described in the previous subsection).

The MOVE system normally presents the main route and its related map elements only. Cross streets are selectively displayed based on their importance to navigating the route. Other map elements such as road labels and landmarks are eliminated if they are not necessary. Renditions can dynamically become important while driving and are selected to be displayed on the screen based on their distance to the vehicle or turns (Figure 15). 


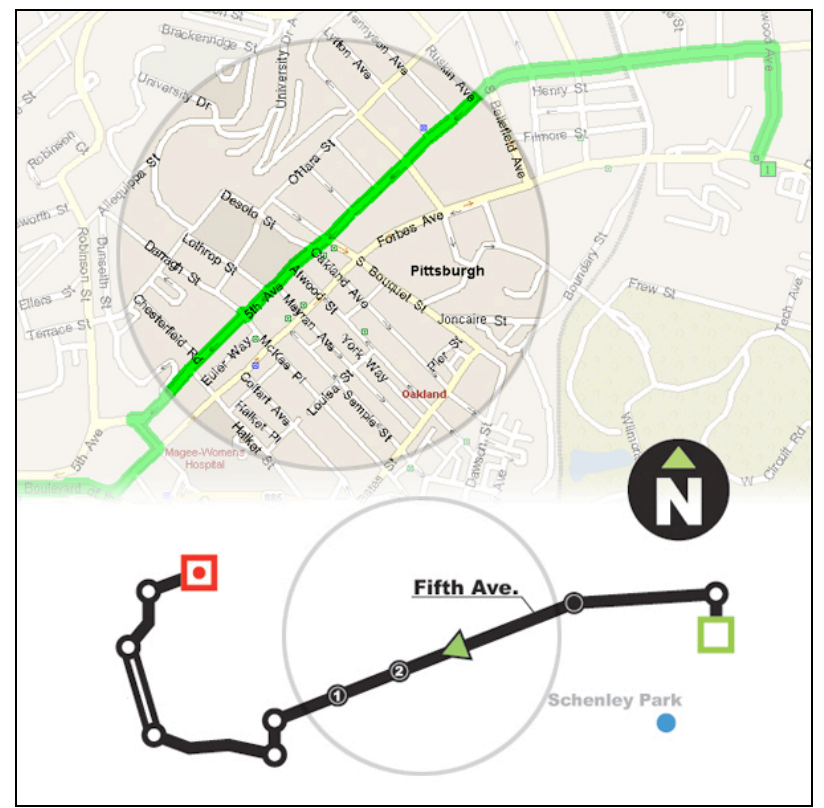

Figure 15. Map feature selection

\subsubsection{Simplification/Smoothing}

Generally, drivers are unaware of a road's actual shape or curvature while driving. For navigation purposes, the shape of the road can be simplified and smoothed in most cases. Presenting the curvature of a road might be important only if it can be used as a milestone or an indication of when and where to turn. This was reflected in our studies, where participants frequently drew maps that distorted the actual curvature of the road (Figure 16).

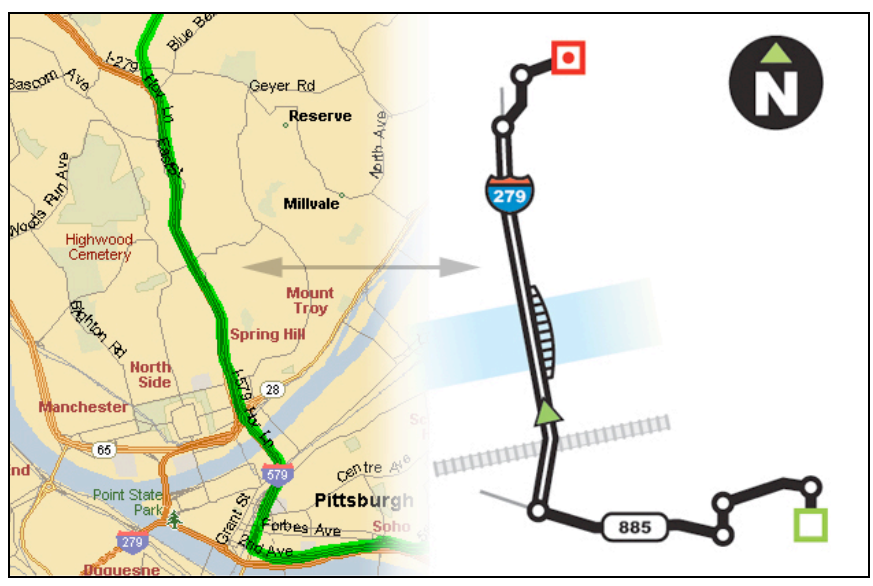

Figure 16. Simplification/Smoothing 


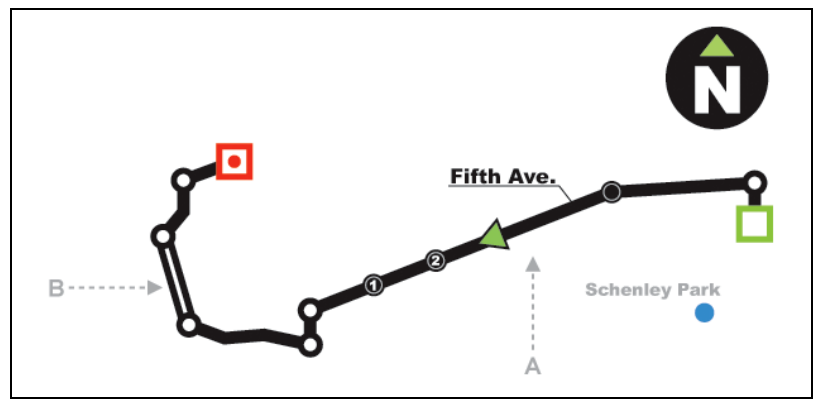

(a)

Figure 17. Relative Scaling, Displacement and Enhancement by map generalization. (a) and (b) are representations of the same route.

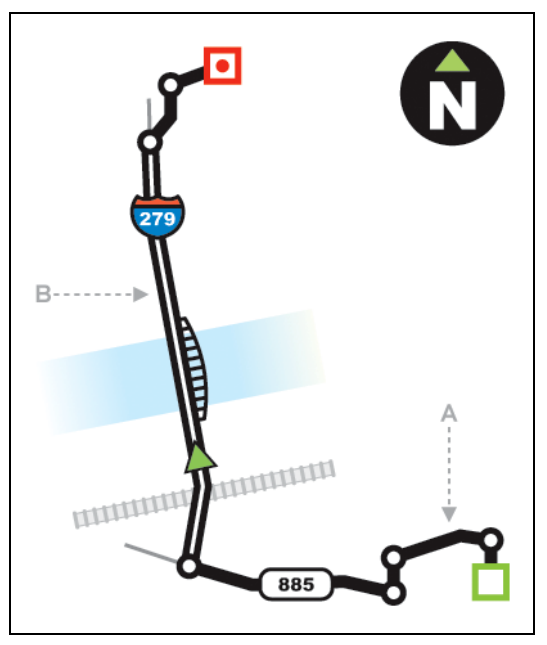

(b)

\subsubsection{Relative Scaling}

The importance of different map features can also be reflected through scaling. The MOVE system will arbitrarily distort the actual road length based on the importance of a segment. The current road segment and segments associated with next turns (regions A and B in Figure 17) are displayed at a larger scale than route segments that are ahead of or behind the driver. In Figure 17a, Route 279 (labeled B) is considerably longer than Fifth Avenue. (labeled A), but Fifth Ave. appears longer because a vehicle is currently traversing Fifth Avenue.

\subsubsection{Displacement}

Labels and renditions that are displayed can possibly interfere with each other. A label might overlap with other labels or renditions, and cross streets labels that are in close proximity might overlap each other when scaling and distortion of the route has taken place. The MOVE system will address such cases by relocating labels and renditions to avoid overlap. For example, in Figure 17a, the road label 'Fifth Ave.' has been relocated in order to avoid overlap with the landmark label 'Schenley Park', and the bridge symbol and river in Figure 17b have been relocated to avoid the railroad tracks.

\subsubsection{Enhancement}

In the right places, detail can enhance navigation. Although many aspects of MOVE displays abstract away detail, enhancement is used when features are important to the current driving context. More detail is applied (primarily through the use of enlarged scale and the selection of additional features) at the final destination of the route, for features associated with the next or current turn, and for features associated with the road segments between the current position and the next turn. For example, as illustrated in Figure $17 \mathrm{a}$, extra cross streets are selected for display when nearing a turn, and these are enhanced with "countdown" number labels indicating how many cross streets are left to pass prior to making the turn.

\subsection{Dynamic Information Interaction}

Our final overarching design principle is dynamic information interaction. Displaying information in the vehicle will present two constraints: screen real estate, and manipulation of the display. Since there is normally very limited screen space available, we cannot put the entire route map within a display. In a traditional in-vehicle navigation system, the driver only sees a small area of the route at once. Typically, scrollbars or navigation buttons are used to access content that is too large for the display. However, such explicit "hands on" interaction is not the most appropriate for the context of driving.

Considerable prior work in information visualization has explored how detail can be rendered in context. Zoomable UIs, "magic" lenses, and fish-eye views are examples of detail-in-context visualizations which 
distort reality to provide detailed information without losing the context of the information (Furnas 1986; Mackinlay, Robertson et al. 1991; Bier, Stone et al. 1993; Bederson and Hollan 1994; Bederson 2000; Bederson, Meyer et al. 2000). With detail-in-context UIs, the user can access detailed information when it is needed, and other contexts that are not important to the user are perceptually minimized (but not removed). All of the content is still accessible at any time.

Research on dynamic information displays has examined the use of time and space as design variables to present bodies of information that are larger than the display area. For example, a dynamic news reader used time, combined with visual cues such as size, color, and emphasis, to present key headlines which faded in importance as time passed (Ishizaki 1996).

To present dynamic navigation information, MOVE accommodates navigation behavior in two ways. First, as learned in our study of navigation, drivers typically break an entire route into sub-goals, focusing on one goal at a time. Therefore MOVE uses the most detail for the road segment that the driver is currently passing over, relative to their goal within the route. Second, the system, using the speed and position of the vehicle, automatically determines which segment to be displayed with detail in context.

Automating the selection of information based on the driver's context will reduce the total attention that the driver needs to expend on a map display. If appropriate information is presented to the driver, the driver's cognitive and attentional loads can be significantly reduced. We can also expect that there would not be any need to physically interact with the display while driving.

To explore dynamic information presentation, we created four different visualization methods as potential candidate for the MOVE system.

\subsubsection{Zoom in Context (ZC)}

In zoom in context (Figure 18), the system automatically enlarges the road segment that the vehicle is passing over to the maximum available size. Other road segments are scaled down to fit on the screen. The advantage of this presentation style is a driver can see the entire route at once, which is useful for getting an overview of the route. However, the vehicle's location cursor moves around the screen inconsistently, so the driver's fixation target is constantly moving.
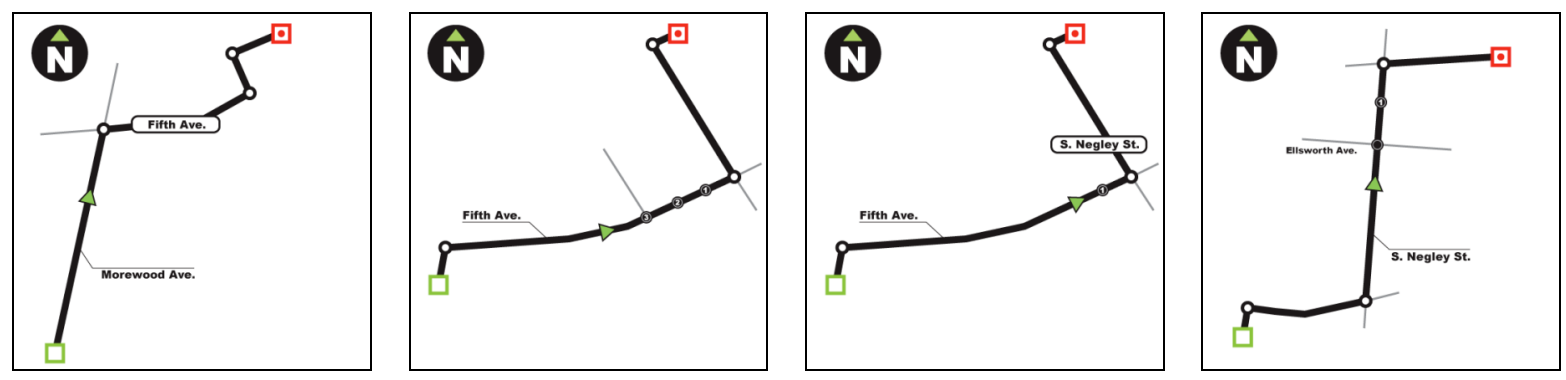

Figure 18. Zoom in Context (ZC)

\subsubsection{Route Scrolling $(R)$}

Route scrolling was developed to overcome the fixation problem described above. In route scrolling, the vehicle's location cursor remains in the center of the screen and the route scrolls as the driver traverses the route (Figure 19). With route scrolling, the driver can easily detect the vehicle's position, but cannot see the entire route at once. Additionally, the route scrolling method does not use screen real estate effectively. Because the vehicle's location cursor remains in the center of the display, one half of the screen is always devoted to part of the route that has already been traversed. 

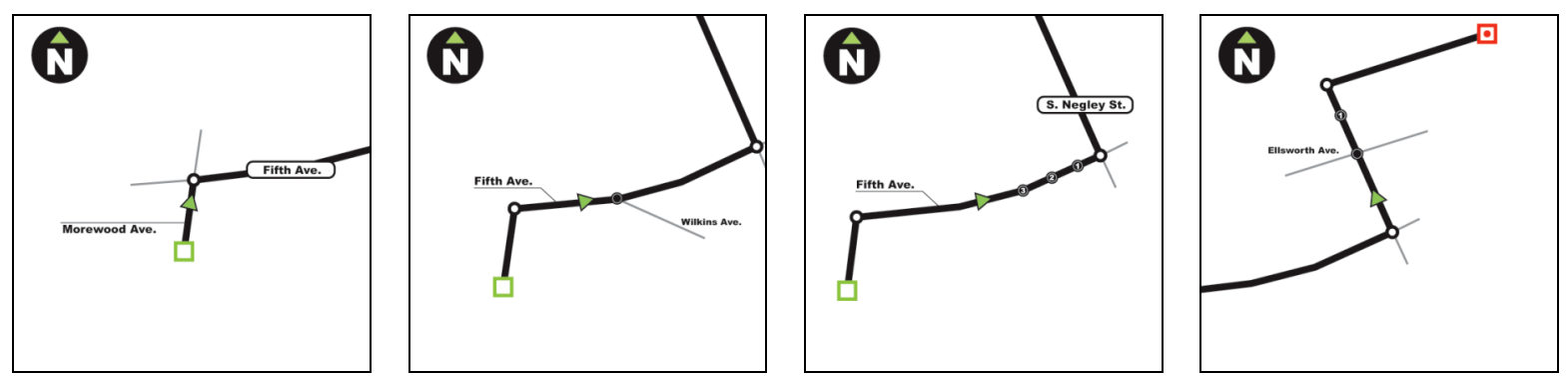

Figure 19. Route Scrolling (R)

\subsubsection{Zoom in Context + Route Scrolling $(Z C+R)$}

To overcome the problems found in zoom in context and route scrolling, we combined the two methods (Figure 20). In this method, the current road segment is displayed at maximum size, while the other segments that have passed scroll from the screen. The driver still can see the overview of the remaining route. However, the driver's fixation target is constantly moving.
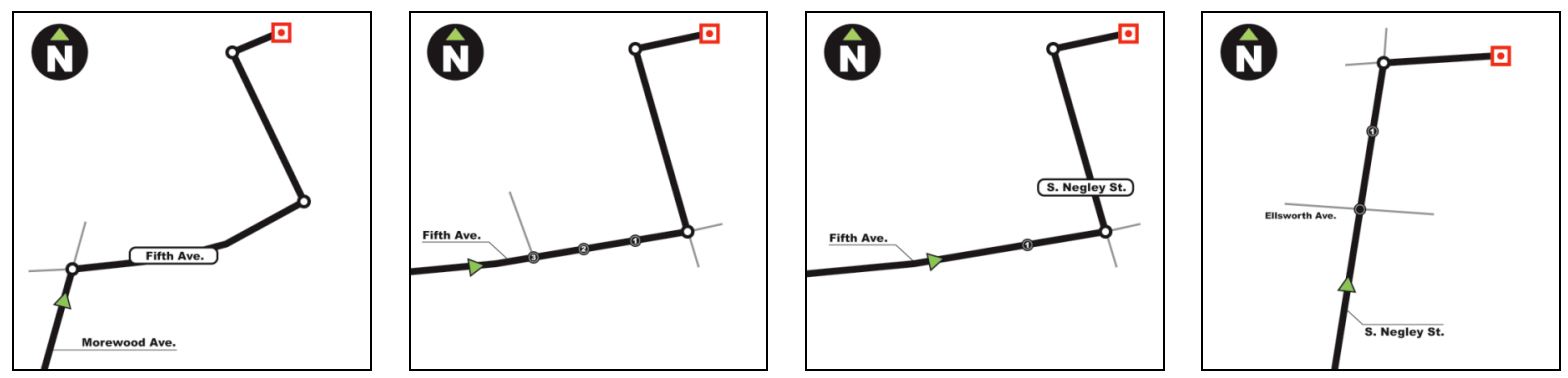

Figure 20. Zoom in Context + Route Scrolling $(\mathrm{ZC}+\mathrm{R})$

\subsubsection{Zoom in Context + Small Overview $(\mathrm{ZC}+\mathrm{O})$}

This method automatically zooms into the section of the route that the driver is currently traversing, while providing a small overview of the route on the lower right of the display (Figure 21). While seeing both the overview and the detail together might be beneficial, the driver will have two areas of focus on the display. This may increase perceptual load while using the system.
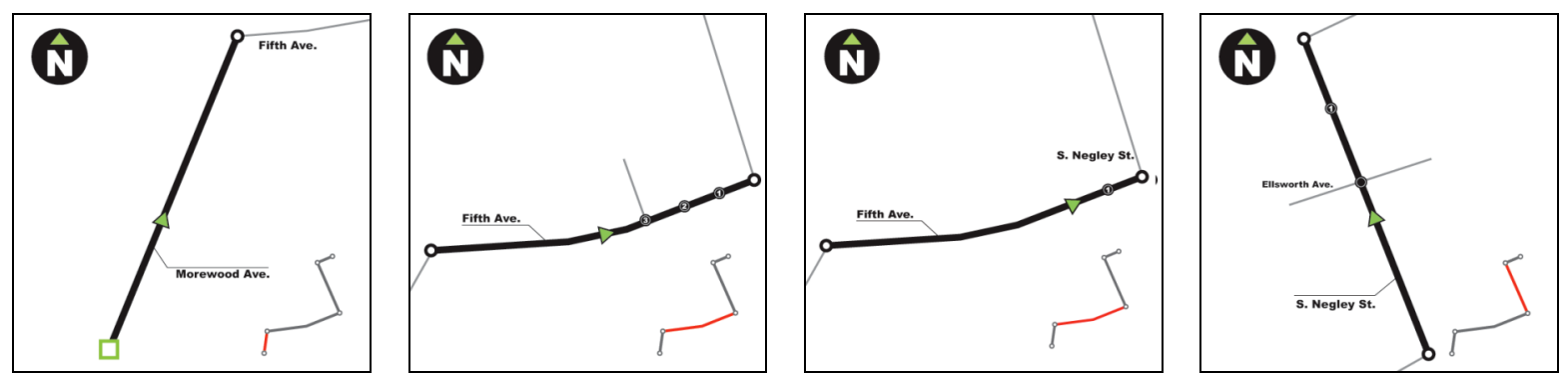

Figure 21. Zoom in Context + Small Overview $(\mathrm{ZC}+\mathrm{O})$

In the next section, we describe a study to evaluate the effectiveness of the four visualization methods and compare it with a static abstract visualization. 


\section{Evaluation and Validation Study}

We conducted a study to evaluate the effectiveness of our prototype design of the MOVE system. The detailed process and results of the study has been reported elsewhere (Lee, Forlizzi et al. 2005), but will be reiterated briefly in this paper for completeness.

The primary purposes of the study were to evaluate the relative merits of our design alternatives and to compare their effectiveness with that of high quality current practice. In addition, we sought to determine whether our prototype of the MOVE system design might satisfy previously developed safety guidelines. The safety guidelines for the design of devices to use while driving have been driven by research on fixation time and number of glances while driving over a number of cultures (Wierwille, Antin et al. 1988; Taoka 1990; Kishi, Sugiura et al. 1992). On average, a driver spends approximately 0.78 seconds (SD = $0.65)$ and 1.26 glances $(\mathrm{SD}=0.40)$ to read a speedometer and 1.10 seconds $(\mathrm{SD}=0.30)$ to check the left mirror. An average of 4.10 seconds fixation time and 2.7 glances on average is the maximum allowed for safely driving a vehicle at $30 \mathrm{~km} / \mathrm{h}$ (VICS 1993). Additionally, Rockwell's Two Second Rule states that drivers do not like to operate a vehicle without roadway information for more than 2 seconds (Rockwell 1988). Guidelines have also been created for the amount of text that can be safely read while driving. Drivers can read an average of 11 Roman characters per second, or 6.2 Japanese characters per second (Ito and Miki 1997; Green 1998).

Clearly, these guidelines show that only a limited amount of information can be presented to a driver at any given time. This is in accordance with our overarching principle that the MOVE system cannot overtax the driver perceptually. If the MOVE system can be designed to reduce the number of glances and fixation times over static maps and current in-vehicle navigation systems, it may very well increase safety while driving.

In the study, we measured the effects of the four MOVE presentation styles described above, along with an extra condition to see the effect of using a cursor. The study was conducted in a lab and LineDrive was used for the baseline comparison. Our hypothesis was that we could reduce the perceptual load of the driver by using the MOVE presentation methods to reduce fixation time and number of glances when interacting with the map. Previous work on in-vehicle navigation guidelines (Rockwell 1988; Dingus and Hulse 1993) have shown that safety of navigation systems can be secured through minimizing the distractions caused by visual information sources other than the road scene. So, our hypothesis was tested by measuring fixation times and numbers of glances of the navigational display in a simple simulated driving task. Our study showed that MOVE's contextually optimized displays do significantly reduce perceptual load. With the contextually optimized displays, total map display fixation time per task averaged $861.98 \mathrm{~ms}$ (compared to an average of $5428.72 \mathrm{~ms}$ for static displays) and average number of glances away from the driving simulator was 1.52 (compared to an average of 4.53 for static displays).

\subsection{Experiment Setup}

Our study used a dual task attention saturating framework, where participants were performing a primary task demanding high levels of attention (using a desktop application reminiscent of driving) and at the same time performing a secondary task (interacting with the navigation display) whose effects on the first task could be measured (Wickens and Hollands 2000). 


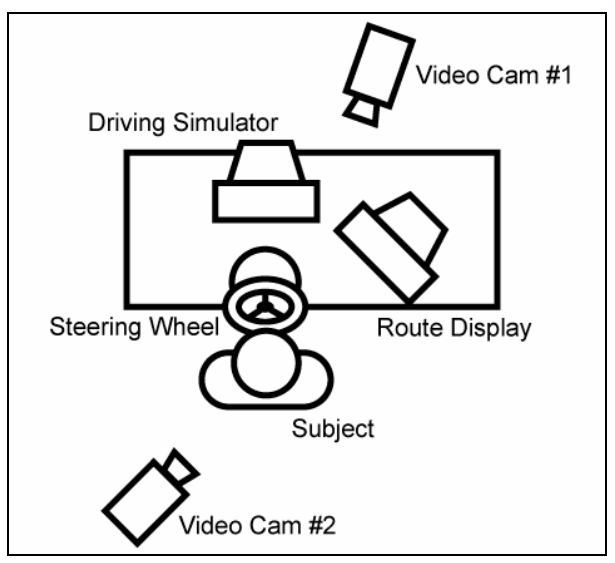

Figure 22. Study configuration

As Figure 22 indicates, we used two displays for the study. The first was placed in front of participants and they performed a simple tracking task in the context of simulated driving. The route scrolled from the top of the screen, and participants were asked to maintain their position in center of the road by moving a red cursor (indicating a car) left or right using a gaming steering wheel input device. We measured the cursor's distance off the road in pixels every $10 \mathrm{~ms}$. This measurement was indicative of whether the primary tracking task was affected by the secondary task. The second display was placed to the right side of the first display. The MOVE display or a static route map was presented. While performing the primary task, the participants were prompted to look at the second display to find answers for the questions specific to the route: for example, "what is your next turn?" or "How many more intersections to the next turn?". Participants then spoke out the answer as soon as they found it from the secondary display. For example, to the questions exemplified above, they might answer "Forbes Avenue" or "Three". During this exercise, total fixation time and number of glances were measured.

Two video cameras were used for data recording. One was placed in front of the participant, capturing eye and head movement. A second camera was placed behind the participant, capturing both displays and a time code.

\subsection{Participants and Procedure}

Twenty participants from the university community aged from 19-56 (12 male and 18 female) participated in the study. All participants completed every condition in counter balanced order.

Before the study session, a baseline trial was performed by every participant. This was to assess each participant's primary task performance. During the study, the four MOVE presentation methods described above were used: $\mathrm{ZC}, \mathrm{ZC}+\mathrm{R}, \mathrm{R}$, and $\mathrm{ZC}+\mathrm{O}$. Each presentation style had four different examples heading north, south, east, and west. LineDrive was used for the static condition baseline comparison. We chose LineDrive because it already reduces visual information significantly compared to traditional maps. LineDrive typography was slightly modified to be more consistent with the typography used in the MOVE designs. In order to isolate the effects of the cursor, we included another ZC condition with the cursor information removed from the display.

\subsection{Results and Discussion}

For the data analysis, we first compared the mean of MOVE with LineDrive (LD) in three measures: Total Fixation Time, Number of Glances, and Average Distance Off the Road. In every measure, MOVE showed better results than $\mathrm{LD}$. MOVE decreased the total fixation time (ms) six-fold $(\mathrm{LD}=5428.72$, MOVE $=861.98$, $t(19)=20.77, p<.0001)$, number of glances threefold $(\mathrm{LD}=4.53$, MOVE $=1.52, t(19)=27.16, p<.0001)$, and average distance off the road (in pixels) five-fold $(\mathrm{LD}=0.0996, \mathrm{MOVE}=0.0204, t(19)=2.304, p=.033)$. All the results were significant at a $5 \%$ significance level. Our main hypothesis was supported by the 
experimental data, suggesting that contextually optimized displays in our MOVE prototype can reduce the driver's perceptual load while navigating

Next, we took a look at the effect of the cursor. When comparing the mean of LD and ZC without cursor, $\mathrm{ZC}$ without cursor performed better than LD in every measure: total fixation time (ms) (LD=5428.72, ZC w/o Cursor $=1049.36, t(19)=18.77, p<.0001)$, number of glances $(\mathrm{LD}=4.53, \mathrm{ZC}$ w/o Cursor=1.76, $t(19)=22.08, p<.0001)$, and average distance off the road (pixel) $(\mathrm{LD}=0.0996, \mathrm{ZC}$ w/o Cursor $=0.0383$, $t(19)=2.872, p=.010)$. However, when we compared $\mathrm{ZC}$ to $\mathrm{ZC}$ without cursor, we saw a small, but statistically significant difference in every measure: total fixation time $(\mathrm{ms})(\mathrm{ZC}=787.17, \mathrm{ZC}$ w/o Cursor=1049.36, $t(19)=5.35, p<.0001)$, number of glances $(\mathrm{ZC}=1.42, \mathrm{ZC}$ w/o Cursor=1.76, $t(19)=5.64$, $p<.0001$ ), and average distance off the road (pixel) $(\mathrm{ZC}=0.0127, \mathrm{ZC}$ w/o Cursor $=0.0383, t(19)=1.300$, $p=.209)$. Even though this effect is much smaller than the six-fold effect of the primary result, this shows that cursor information is helpful in locating information.

The reason may be because when reading the static map, participants actually performed two tasks: searching for context and then finding needed information. Within the contextually optimized display, even though there was no cursor to give specific location information, orientation to the context helped participants to reduce search time.

Finally we compared our four presentation methods. ZC was used for baseline comparison. $\mathrm{ZC}$ and $\mathrm{ZC}+\mathrm{R}$ showed better performance in every measure. However, there was no significant difference in the measure of average distance off the road. There was also no significant difference found between $\mathrm{ZC}$ and $\mathrm{ZC}+\mathrm{R}$.

This is possibly because the $\mathrm{R}$ and $\mathrm{ZC}+\mathrm{O}$ designs are less effective. The $\mathrm{R}$ style lacks the zoom-in-context feature, which is critical for quickly finding information. Additionally, $\mathrm{R}$ uses only one quarter of the screen for presenting important information (Figure 23). The $\mathrm{ZC}+\mathrm{O}$ style has two information sources, which results in complexity and the need to perceive two information sources at once. Additionally, the small overview in this style sometimes overlaps the larger route rendition. This creates more complexity, as well as not using screen real estate effectively.

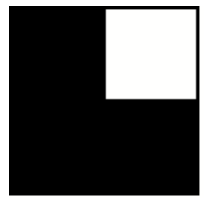

$\mathrm{R}$

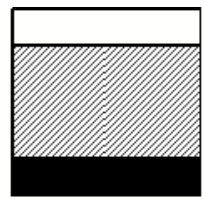

$\mathrm{ZC}+\mathrm{O}$ $\square$ Most frequently used

Usilg Used sometimes

Not used

\section{Figure 23. Use of screen real estate in $\mathrm{R}$ and $\mathrm{ZC}+\mathrm{O}$. Only small part of the screen was used for} information display.

However, this study did not look specifically at navigation performance of our display. The study was rather focused purely on the efficiency of map reading, since prior studies (Rockwell 1988; Dingus and Hulse 1993) have indicated that reducing the visual attention to the map display could improve driving safety. As a result, it is possible that the study result might not translate to actual navigation behavior. However, we have shown that our display can substantially reduce a driver's attention to the display. A navigation performance study with our MOVE display is planned for future work.

\section{Conclusion and Future Work}

This paper has presented our iterative design and evaluation of the MOVE system. Initial research on navigation showed that abstracted and simplified route representations support the way people use maps. A subsequent study on visual search revealed how different map renditions can attract attention when designing a particular route. Semantic renditions should be used for important target areas, while symbolic renditions can be used for less-important areas. Renditions inducing perceptual pop-outs should be reserved for the most important aspects of the display. 
We next developed design principles and used them to design four prototype presentation styles of the MOVE system. A lab study evaluating the MOVE prototypes shows that contextually optimized displays should significantly decrease the driver's perceptual load caused by visual map information during the context of driving. We found large decreases in the total fixation time and the number of glances comparing the contextually optimized display to a static display. We also found that driving performance was less affected with the contextually optimized route displays. Additionally, the study revealed the best possible design alternatives for the MOVE system: zoom in context (ZC) and zoom in context + route scrolling $(\mathrm{ZC}+\mathrm{R})$ were selected because of the best use of screen real estate.

Even though these results are very promising, we still need further validation of the system in various conditions. First, since our evaluation study was performed with a low fidelity driving simulator, we were not able to directly measure navigation performance with our system. So, we need further study on this. Second, due to the limit of lab-based study, we couldn't test which style (heading-up vs. north-up) is preferred in the dynamically optimized map display. Prior study (Dingus and Hulse 1993; Mashimo, Daimon et al. 1993; Ross, Vaughan et al. 1995) has indicated that heading-up is preferred when driving while north-up is more efficient when trip planning. We need to look into whether this result is still applicable for our contextually optimized display. Currently we are implementing the system to be used in an actual vehicle. Our next steps will define clear methods for optimizing map visual information and then test navigation performance with the prototype of MOVE system. As Denis (Denis, Pazzaglia et al. 1999) previously indicated, appropriately selected map features and route map with good descriptions could enhance navigation performance. In our next study, we will examine whether our optimization algorithm could successfully generate a route map that accomplishes this.

\section{Acknowledgements}

This work was supported in part by the National Science Foundation under grants IIS-0121560 and IIS0325351, in part by funding from the General Motors Corporation, and this material is based in part upon work supported by the Defense Advanced Research Projects Agency (DARPA) under Contract No. NBCHD030010.

\section{References}

Agrawala, M. and C. Stolte (2001). Rendering Effective Route Maps: Improving Usability through Generalization. ACM SIGGRAPH 2001, Los Angeles, CA, ACM Press.

Baldassi, S. and D. C. Burr (2004). "'Pop-out" of targets modulated in luminance or colour: the effect of intrinsic and extrinsic uncertainty." Vision Research 44: 1227-1233.

Beck, J. (1982). Textural segmentation. Organization and representation in perception. J. Beck. Hillsdale, NJ, Erlbaum.

Bederson, B. B. (2000). Fisheye Menus. ACM UIST 2000, San Diego, CA, ACM Press.

Bederson, B. B. and J. D. Hollan (1994). Pad++: A Zooming Graphical Interface for Exploring Alternate Interface Physics. ACM UIST 1994, Marina Del Ray, CA, ACM Press.

Bederson, B. B., J. Meyer, et al. (2000). Jazz: an extensible zoomable user interface graphics toolkit in Java. ACM UIST 2000, San Diego, CA, ACM Press.

Bier, E. A., M. C. Stone, et al. (1993). Toolglass and Magic Lenses: The See-through Interface. ACM SIGGRAPH 1993, Anaheim, CA, ACM Press.

Burnett, G. E. (1998). "Turn Right at the King's Head": Drivers' Requirements for Route Guidance Information. UK, Loughborough University. P h. D.

Burnett, G. E. (2000). "Turn Right at the Traffic Lights: The Requirements for Landmarks in Vehicle Navigation systems." The Journal of Navigation 53(3): 499-510.

Burnett, G. E., S. J. Summerskill, et al. (2004). "On-the-move destination entry for vehicle navigation systems: Unsafe by any means?" Behaviour and Information Technology 23(4): 265-272.

Conner, C. E., H. E. Egeth, et al. (2004). "Visual Attention: Bottom-Up Versus Top-Down." Current Biology 14: R850-R852.

Council, V. P. (1993). VICS Promotion Council Safety Committee Report on Information Display. Tokyo, Japan, VICS Promotion Council. 
Denis, M., F. Pazzaglia, et al. (1999). "Spatial Doscourse and Navigation: An Analysis of Route Directions in the City of Venice." Applied Cognitive Psychology 13: 145-174.

Dingus, T. A. and M. C. Hulse (1993). "Some Human Factors Design Issues and Recommendations for Automobile Navigation Information Systems." Transportation Research 1C(2): 119-131.

Furnas, G. W. (1986). Generalized Fisheye Views. ACM CHI 1986, Boston, MA, ACM Press.

Goldstein, E. B. (2002). Sensation and Perception. Pacific Grove, CA, Wadsworth.

Golledge, R. G. (1999). Wayfinding Behavior: Cognitive Mapping and Other Spatial Processes. Baltimore, MD, Johns Hopkins University Press.

Green, P. (1998). Visual and Task Demands of Driver Information Systems. Ann Arbor, MI, The University of Michigan Transportation Research Institute.

Ishizaki, S. (1996). Multiagent Model of Dynamic Design: Visualization as an Emergent Behavior of Active Design Agents. ACM CHI 1996, Vancouver, Canada, ACM Press.

Ito, T. and Y. Miki (1997). Japan's Safety Guideline on In-Vehicle Display Systems. Proceedings of the Fourth ITS World Congress, Brussels, Belgium, VERTIS.

Julesz, B. (1984). "A brief outline of the texton theory of human vision." Trends in Neuroscience 7: 41-45.

Kishi, H., S. Sugiura, et al. (1992). "Visibility Considerations for Automobile Navigation Displays (in Japanese)." Jidosha Gijutsu (Journal of the Society of Automotive Engineers of Japan) 46(9): 6167.

Labiale, G. (1990). In-Car Road Information: Comparisons of Auditory and Visual Presentation Proceedings of the Human Factors and Ergonomics Society 34th Annual Meeting, Santa Monica, CA, Human Factors and Ergonomics Society.

Lee, J., J. Forlizzi, et al. (2005). Studying the Effectiveness of MOVE: A Contextually Optimized InVehicle Navigation System. ACM CHI 2005, Portland, OR, ACM.

Llaneras, R. E. and J. P. Singer (2003). In-Vehicle Navigation Systems: Interface Characteristics and Industry Trends. Driving Assessment 2003, 2nd International Driving Symposium on Human Factors in Driver Assessment, Training and Vehicle Design.

MacEachren, A. M. (1995). How Maps Work : Representation, Visualization, and Design. New York, NY, Guilford Press.

Mackinlay, J. D., G. G. Robertson, et al. (1991). The Perspective Wall: Detail and Context Smoothly Integrated. ACM CHI 1991, New Orleans, LA, ACM Press.

Mashimo, S., T. Daimon, et al. (1993). Drivers' characteristics for map information representation (North up map/heading up map) in navigation displays. Proceedings of Vehicle Navigation and Information Systems Conference, Piscataway, NJ, Institute of Electrical and Electronics Engineers.

Michon, J. A. (1985). A Critical View of Driver Behaviour Models. Human Behaviour and Safety. L. Evans and R. S. Schwing. New York, Plenum Press: 485-524.

Michon, J. A., Ed. (1993). Generic Intelligent Driver Support: A comprehensive report on GIDS. London, Taylor and Francis.

Microsoft (2005). MapPoint, http://mappoint.msn.com.

Monmonier, M. (1996). How to Lie with Maps. Chicago, IL, The University of Chicago Press.

Parks, A. M., M. C. Ashby, et al. (1991). The Effect of Different In-Vehicle Route Information Displays on Driver Behavior. Vehicle Navigation and Information Systems Conference Proceedings, Warrendale, PA, Society of Automotive Engineers.

Rockwell, T. H. (1988). Spare Visual Capacity in Driving - Revisited: New Empirical Results of an Old Idea. Vision in Vehicles II, Amsterdam, Elsevier Science B.V. (North-Holland).

Ross, T. and G. E. Burnett (2001). "Evaluation of the Human-Machine Interface to Vehicle Navigation Systems as an Example of Ubiquitous Computing." International Journal of Human-Computer Studies 55(4): 661-674.

Ross, T., G. Vaughan, et al. (1995). Human factors guidelines for information presentation by route guidance and navigation systems. DRIVE II V2008 HARDIE, Deliverable 19. Loughborough, UK, HUSAT Research Institute.

Streeter, L. A., D. Vitello, et al. (1985). "How to Tell People Where to Go: Comparing Navigational Aids." International Journal of Man-Machine Studies 22(5): 549-562.

Taoka, G. T. (1990). "Duration of Drivers' Glances at Mirrors and Displays." Institute of Transportation Engineers Journals 60(10): 35-39. 
Tijerina, L., E. Parmer, et al. (1998). Driver Workload Assessment of Route Guidance System Destination Entry While Driving: A Test Track Study. Ann Arbor, MI, The University of Michigan Transportation Research Institute.

Treisman, A. (1986). "Features and obects in visual processing." Scientific American 255: 114B-125B.

Treisman, A. (1998). The perception of features and objects. Visual attention. R. D. Wright. New York, Oxford University Press: pp. 26-54.

Wickens, C. D. and J. G. Hollands (2000). Engineering Psychology and Human Performance. Upper Saddle River, NJ, Prentice Hall.

Wierwille, W. W., J. F. Antin, et al. (1988). Visual Attentional Demand of an In-Car Navigation Display

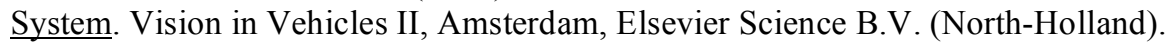

